\title{
Neural basis of shame and guilt experience in women with borderline personality disorder
}

\author{
Martin Göttlich ${ }^{2}$ Anna Lisa Westermair ${ }^{1} \cdot$ Frederike Beyer $^{4} \cdot$ Marie Luise Bußmann $^{1}$ - Ulrich Schweiger ${ }^{1}$. \\ Ulrike M. Krämer ${ }^{2,3}$ (1)
}

Received: 30 November 2019 / Accepted: 27 April 2020 / Published online: 7 May 2020

(c) The Author(s) 2020

\begin{abstract}
Borderline personality disorder (BPD) is characterized by instability of affect, emotion dysregulation, and interpersonal dysfunction. Especially shame and guilt, so-called self-conscious emotions, are of central clinical relevance to BPD. However, only few experimental studies have focused on shame or guilt in BPD and none investigated their neurobiological underpinnings. In the present functional magnetic resonance imaging study, we took a scenario-based approach to experimentally induce feelings of shame, guilt, and disgust with neutral scenarios as control condition. We included 19 women with BPD (age 26.4 \pm 5.8 years; DSM-IV diagnosed; medicated) and 22 healthy female control subjects (age 26.4 \pm 4.6 years; matched for age and verbal IQ). Compared to controls, women with BPD reported more intense feelings when being confronted with affective scenarios, especially higher levels of shame, guilt, and fear. We found increased amygdala reactivity in BPD compared to controls for shame and guilt, but not for disgust scenarios ( $p=0.05 \mathrm{FWE}$ corrected at the cluster level; $p<0.0001$ cluster defining threshold). Exploratory analyses showed that this was caused by a diminished habituation in women with BPD relative to control participants. This effect was specific to guilt and shame scenarios as both groups showed amygdala habituation to disgust scenarios. Our work suggests that heightened shame and guilt experience in BPD is not related to increased amygdala activity per se, but rather to decreased habituation to self-conscious emotions. This provides an explanation for the inconsistencies in previous imaging work on amygdala involvement in BPD as well as the typically slow progress in the psychotherapy of dysfunctional self-conscious emotions in this patient group.
\end{abstract}

Keywords Borderline personality disorder $\cdot$ Self-conscious emotions $\cdot$ Shame $\cdot$ Guilt $\cdot$ Amygdala

Communicated by Sebastian Walther.

Martin Göttlich and Anna Lisa Westermair contributed equally to this work.

Electronic supplementary material The online version of this article (https://doi.org/10.1007/s00406-020-01132-z) contains supplementary material, which is available to authorized users.

Ulrike M. Krämer

ulrike.kraemer@uni-luebeck.de

1 Department of Psychiatry and Psychotherapy, University of Lübeck, Lübeck, Germany

2 Department of Neurology, University of Lübeck, Ratzeburger Allee 160, 23538 Lübeck, Germany

3 Institute for Psychology II, University of Lübeck, Lübeck, Germany

4 School of Biological and Chemical Sciences, Queen Mary University of London, London, UK

\section{Introduction}

Borderline personality disorder (BPD) is defined by a pervasive pattern of instability in interpersonal relationships, selfimage, and affect, as well as markedly impulsive behavior [1]. It is associated with a high rate of comorbidity (above $80 \%$ ), severe psychosocial impairment, intensive use of the health care system, and a high risk of suicide (5-10\%) [2, $3]$. It is also relatively common with a point prevalence of $1 \%$ in the general population, $12 \%$ in outpatient, and $22 \%$ in inpatient mental health care [4]. The various conceptualizations of this complex disorder all include emotion dysregulation, interpersonal sensitivity, and difficulties with social cognition [5].

As they touch on all these deficits, BPD patients show particularly pronounced levels of the self-conscious emotions shame and guilt $[6,7]$. Both are negative affective states following a shortcoming or transgression in an 
interpersonal situation that is attributed internally [8]. In the case of shame, the attribution is global and stable, resulting in a negative evaluation of the global self ("I did something bad!"). Shame is generally described as feeling small, worthless, and exposed $[9,10]$ and leads to rumination about the self and personal distress [11]. The corresponding action tendencies are to deny, hide, or escape the shame-inducing situation, e.g. by externalization of blame. This typically leads to intense anger that is expressed destructively [10, $12,13]$, thus, shame-proneness is negatively associated with pro-social orientation [8].

In the case of guilt, the transgression is attributed specifically and instable, meaning the focus is on the problematic act and the social other ("I did something bad to them!") [14-16]. Guilt increases self-reflection and perspective taking, and facilitates reparative behaviors such as confessing, apologizing, and making amends $[10,11]$. Therefore, guiltproneness is associated with pro-social orientation and positive relationship outcomes $[8,17]$ and has been identified as a component of trait morality [18]. Shame-proneness is associated with a wide range of dysfunctional behavior (such as deliberate self-harm, substance abuse, and risky sexual behavior) and mental health problems (including depression, eating disorder symptoms, and suicidal ideation), whereas the propensity to experience "shame-free" guilt is not $[10$, 19, 20]. Patients with BPD experience higher levels of state and trait shame than both healthy controls and patients with other axis I or axis II disorders [21-24], whereas guilt correlates negatively with BPD symptoms [25].

Despite the clinical relevance of self-conscious emotions and evidence from questionnaire data speaking for altered shame and guilt experience in BPD, only little experimental work has been conducted on this topic. Gratz et al. [22] found elevated and prolonged feelings of shame in BPD outpatients after a laboratory stressor. This shows that intensified experience of shame in BPD also pertains to experimental situations. Studying shame and guilt in lab experiments poses some challenges. As the attributional style and the self-concept of an individual influences which social emotion is elicited, it varies between subjects [26]. For instance, experiencing an unwanted mishap might trigger embarrassment in some persons, whereas it might be attributed to oneself as a person and thus elicit shame in others. This seems particularly challenging when studying BPD. Failure in achievement situations might be less central to the self-concept of many BPD patients compared to controls [27]. On the other hand, autobiographical memory paradigms might trigger traumatic experiences in persons with BPD more so than in others [28]. In the current study, we took a scenariobased approach. The scenarios all described interpersonal situations, were designed to avoid triggering flashbacks and did not involve any achievement- or job-related situations (see "Methods" for further description).
These scenarios were used to explore the underlying neural correlates of intensified social emotions in BPD. Evoking shame and guilt within the constraints of MRI is even more challenging than in behavioral studies, as the MRI setting heavily constrains actual personal interactions in which social emotions could evolve [26]. The scenariobased paradigm in the current study was designed to experimentally induce feelings of shame, guilt, and disgust and compare them to neutral scenarios. In previous studies using this approach $[29,30]$, the authors reported activation especially in medial prefrontal areas and superior temporal areas. In addition, experience of guilt elicited insula activation, whereas experience of shame elicited amygdala activity in persons with remitted major depression [31]. A recent review on the neural correlates of shame, embarrassment, and guilt [32] concluded that these social emotions are associated with distinct yet overlapping neural networks, but that results are quite heterogeneous across studies which necessitates further research.

No previous imaging study addressed shame and guilt experience in BPD, but numerous fMRI studies shed light on the neural underpinnings of altered emotional experience and emotion regulation deficits in persons suffering from BPD [33]. Based on the emotion dysregulation theory of BPD [34], many of these studies focused on the amygdala, reporting both increased and decreased activity while processing emotional stimuli $[33,35]$. Explanations for these inconsistencies include sample characteristics (small sample size, comorbidities), task methodology (missing emotionally neutral condition), and psychological processes such as habituation or dissociation [33, 35]. Hazlett et al. [36] reported a potentiated amygdala response to repeatedly shown affective pictures in BPD. Patients exhibited normal amygdala activation during novel pictures but relatively increased amygdala activation during repeated pictures compared with $\mathrm{HC}$ which supports the notion of altered habituation in BPD.

The complex symptomatology of BPD clearly goes beyond a mere dysfunction of the amygdala. Evidence exists for altered activity in regions implicated in social cognition, empathy, and self-referential processing including the insula, medial PFC, superior temporal sulcus, and precuneus [33]. As mentioned above, several of these regions have also been found in fMRI studies on social emotions, suggesting that dysfunction within this network might be related to elevated levels of shame experience in BPD.

In the present study, we primarily aimed to investigate the neural correlates of elevated shame and guilt experience in women diagnosed with BPD, especially in the above-mentioned networks of social cognition, empathy, and self-referential processing. A secondary goal was to test for correlations between shame/guilt related brain reactivity and disease severity as measured by the borderline symptom 
inventory (BSI). Motivated by previous experimental evidence of a reduced decline in shame experience over time in BPD patients [22] and theoretical considerations of delayed habituation effects in BPD [34], we explored the temporal evolution of amygdala reactivity during repeated exposure to shame and guilt scenarios.

Addressing the criticisms towards earlier studies reporting partially contradicting findings as discussed above, we included 19 severely affected BPD patients with typical psychiatric comorbidity and 22 healthy controls and included both an emotionally neutral condition and a non-social emotional condition (disgust). We reduced the risk of dissociation by (i) omission of potentially flashback-triggering scenario content, (ii) allowing for familiarization with the study personnel and MRI setting prior to BOLD measurements, and (iii) monitoring of inner tension between runs. Given the heterogeneity of previous imaging results in BPD and on social emotions, we took a whole-brain analysis approach to investigate altered activity for social emotions and specifically for shame experience. To avoid false positives, we controlled the family-wise error rate in our whole-brain analyses. We expected to find enhanced subjective experience of shame in BPD patients and aberrant activations in the amygdala, and brain regions implicated previously in social emotions, namely the medial prefrontal cortex, the anterior insula, precuneus, and inferior frontal gyrus [32].

\section{Methods}

\section{Participants}

All subjects were female, right-handed (self-reported), had German as their native language, were 18-35 years of age, and had normal or corrected to normal vision. Men were excluded in order to gain a sufficiently homogeneous sample. Exclusion criteria for both groups were a history of a central nervous system disease or major head trauma, unwillingness to refrain from alcohol consumption one week prior to each study appointment as well as all MR contraindications (e. g. ferromagnetic or electronic implants).

We recruited 28 women with borderline personality disorder (BPD) at the Department of Psychiatry and Psychotherapy, University of Lübeck, Germany, upon admission to an open ward for inpatient dialectical behavior therapy. All patients were assessed using the German version of the Structured Clinical Interview for DSM-IV axis I and axis II disorders [37]. All women with BPD met criteria for at least one axis I disorder with mood, posttraumatic stress, and eating disorders being the most frequent (Table 1), consistent with other studies [38]. Verbal IQ was measured with a multiple choice vocabulary test (Mehrfachwahl-WortschatzIntelligenz test, MWT-B) [39]. The MWT-B is an economic measure of verbal intelligence widely used in psychological research due to its well-established correspondence to more detailed test batteries such as HAWIE-R [40]. Exclusion criteria for the BPD group were a history of schizophrenia, addiction or mental retardation; acute metabolic disturbance (e.g. low potassium), and recent medication with or abuse of benzodiazepines, opioids or alcohol. Other psychotropic medication had to be in steady state at the time of MR measurements and is shown in Supplementary Table 1. From the 28 women with BPD originally recruited to the study, four dropped out for medical reasons (e.g. ingestion of metallic objects), four for personal reasons, and one due to excessive head motion. Their data was not included in the analysis. 13 of the 19 women in the final BPD sample had undergone more than one year of (inpatient or outpatient) psychotherapy prior to recruitment for this study, of which three had undergone more than 5 years.

As controls, 32 healthy women were recruited via local online advertisement and black boards at the local blood donation center, university medical center, public library, and supermarkets. They were matched to age and verbal IQ of the patient group and carefully screened for any current or lifetime axis I or II disorder using the above-mentioned interviews. From the 32 healthy women originally recruited to the study, four dropped out for personal reasons, four for medical reasons (abnormalities detected in structural MR images), and two for excessive head motion. Their data were removed from the analysis, leaving 22 women in the control group.

All participants gave written informed consent after the study protocol had been carefully explained to them. Participants who completed the study received their structural MR images on DVD, $30 €$, and a summary of the study's results in layman terms per email. The study was designed in line with the Declaration of Helsinki and approved by the local ethics committee prior to recruitment (AZ 12-080). Demographic and clinical data of the final study population are presented in Table 1 and Supplementary Table 1. There was no difference between groups regarding age, weight, or verbal IQ. Groups differed with regard to severity of BPD symptoms, handedness, and level of education.

\section{Experimental paradigm}

Participants were presented with short descriptions of fictitious scenarios carrying either shame-, guilt-, disgustrelated, or neutral content. The disgust condition served as negative control condition without social aspect. All scenarios were presented in one German sentence written in second-person, present tense (see Supplementary Table 2 for English translations). Scenarios were selected based on theoretical considerations: as their disorder prevents many BPD patients from working, this setting was excluded. Family and 
Table 1 Clinical and sociodemographic characteristics of the sample

\begin{tabular}{|c|c|c|c|}
\hline & $\begin{array}{l}\text { Women with } \\
\operatorname{BPD}(N=19)\end{array}$ & $\begin{array}{l}\text { Healthy women } \\
(N=22)\end{array}$ & Test statistic \\
\hline Age (years) & $26.5(5.8)$ & $26.4(4.6)$ & $t(39)=-0.68, p=0.946$ \\
\hline Verbal IQ & $94.8(6.6)$ & $96.4(6.5)$ & $t(39)=0.74, p=0.462$ \\
\hline BMI $\left(\mathrm{kg} / \mathrm{m}^{2}\right)$ & $28.7(10.0)$ & $24.6(5.0)$ & $t(25.5)=-1.62, p=0.118$ \\
\hline Handedness (lateralization quotient) ${ }^{\mathrm{a}}$ & $87.5(9.9)$ & $76.9(17.7)$ & $t(38)=-2.53, p=0.030$ \\
\hline \multicolumn{4}{|l|}{ Secondary education } \\
\hline None & 0.0 & $4.5^{\mathrm{d}}$ & $\gamma=-0.46, p=0.047$ \\
\hline Grundschule (graduation after 4 years) & 5.3 & 0.0 & \\
\hline Hauptschule (8 years) & 26.3 & 4.5 & \\
\hline Realschule (9 years) & 42.1 & 40.9 & \\
\hline Abitur (13 years) & 26.3 & 59.0 & \\
\hline Currently employed & 47.4 & 63.6 & $\chi^{2}(1)=1.10, p=0.295$ \\
\hline \multicolumn{4}{|l|}{ Mental disorders } \\
\hline Any axis I disorder & 100.0 & & \\
\hline Mayor depressive episode & 63.2 & & \\
\hline Social phobia & 5.3 & & \\
\hline Panic disorder & 15.7 & & \\
\hline PTSD & 36.8 & & \\
\hline OCD & 10.5 & & \\
\hline ADHD & 10.2 & & \\
\hline Any eating disorder & 73.7 & & \\
\hline Other personality disorder & 10.5 & & \\
\hline \multicolumn{4}{|l|}{ Questionnaire data } \\
\hline Borderline symptom inventory $(\mathrm{BSI})^{\mathrm{b}}$ & $33.4(6.7)$ & $1.0(1.5)$ & $Z^{\mathrm{e}}=-5.53, p<0.001$ \\
\hline Shame-proneness ${ }^{c}$ & $42.7(8.3)$ & $24.5(8.5)$ & $t(39)=-6.95, p<0.001$ \\
\hline Guilt-proneness $^{c}$ & $48.7(5.9)$ & $42.0(5.6)$ & $t(39)=-3.78, p<0.001$ \\
\hline Detachment-proneness ${ }^{\mathrm{c}}$ & $22.3(4.3)$ & $29.4(5.4)$ & $t(39)=4.60, p<0.001$ \\
\hline External-attribution-proneness ${ }^{\mathrm{c}}$ & $20.1(5.0)$ & $21.2(5.0)$ & $t(39)=0.71, p=0.481$ \\
\hline
\end{tabular}

PTSD posttraumatic stress disorder, $O C D$ obsessive-compulsive disorder, $A D H D$ attention deficit and hyperactivity disorder

${ }^{a}$ Measured with the Edinburgh Inventory [61]

${ }^{\mathrm{b}}[45]$

${ }^{c}$ Measured with the TOSCA-3 questionnaire [42]

${ }^{\mathrm{d}}$ These controls were still attending school. Numbers refer to mean and standard deviation in brackets or percentage values (for secondary education and mental disorders)

${ }^{\text {e}}$ Test statistic of the Mann-Whitney $U$ test relationship settings were omitted to avoid triggering flashbacks. Also, we opted against an individualized approach (e.g. using personal memories) as this would probably result in women with BPD imagining more severe and qualitatively different situations (e.g. sexual abuse) than healthy women, in whom the worst shame-associated memory might be losing one's head during an oral presentation [26]. Instead, we focused on body shame, as it is of high relevance to the clinical management of BPD and has been linked to both childhood abuse and eating disorder symptoms in adulthood [10]. Scenarios were adapted according to results of penand-pencil pilot studies with 32 healthy volunteers and six women with BPD (see Supplementary Table 3 for results of the pilot study).
Scenarios were programmed and presented with Presentation $^{\mathrm{TM}}$ (Neurobehavioral Systems, Albany, N.Y., USA), and displayed via VisuaStim Digital ${ }^{\mathrm{TM}}$ goggles. In each trial (Fig. 1a), the scenario was presented for $9 \mathrm{~s}$, followed by the German word for "Imagine" for $20 \mathrm{~s}$, followed by a distraction task (pressing a button when the number 3 was presented) for $16 \mathrm{~s}$. In the case of two controls, distraction task data were lost due to technical error. Each run contained the same 12 scenarios ( 3 scenarios for each condition) in changing, pseudorandomized order; the experiment comprised three runs with a total duration of $30 \mathrm{~min}$. Inner tension was reported orally by participants between runs on a scale from 0 to 100 . This form of verbalization is an integral part of the dialectic behavioral psychotherapeutic concept 
A

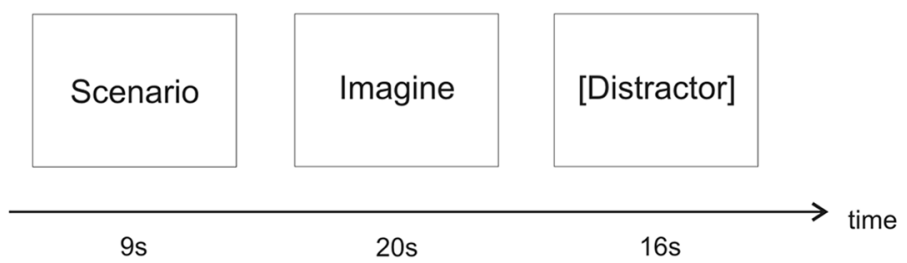

B

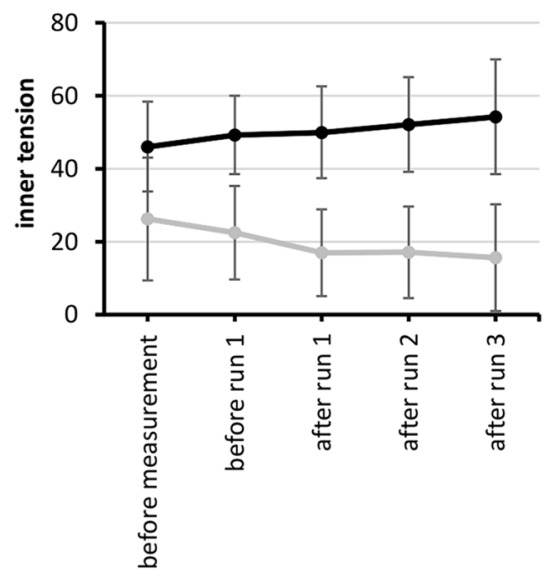

C

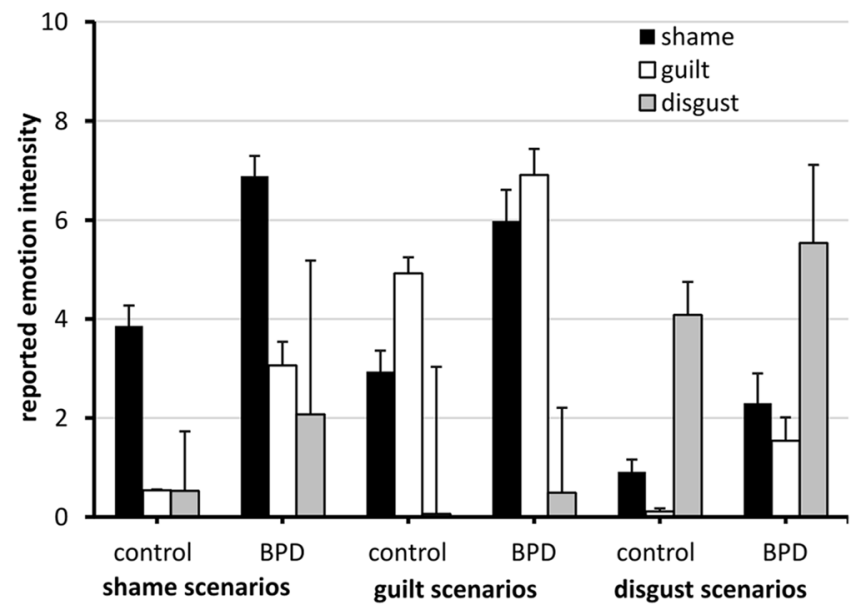

Fig. 1 Trial outline and behavioral data. a Time-line of one individual trial. b Self-reported inner tension over the course of the BOLD measurement in control group (gray line) and BPD group (black line).
Error bars indicate one standard deviation. For test statistics see text. c Self-reported intensity of shame, guilt and disgust for the three scenario types. Error bars indicate one standard deviation of the open ward we recruited patients from [41]. If patients indicated inner tension level of 100, measurement was to be halted and patients guided through stress resistance skills. This was not necessary in any case. Directly after scanning, participants rated every scenario on 10-point Likert scales regarding vividness of imagination and intensity of their emotional response.

\section{Study procedures}

To level out differences in prior MR experience minimizing emotional responses to the study setting as a potential confounding factor, and to allow for familiarization with the MR setting in order to reduce the risk of dissociation, MR measurements were divided into two appointments, with anatomical and DTI measurements in the first and BOLD measurements of the experimental paradigm in the second. Participants met with the same member of the study team at all appointments, also in order to allow for familiarization. Participants additionally filled out questionnaires: The test of self-conscious affect-3 (TOSCA-3) [42] is a self-report questionnaire using social scenarios to assess proneness to social emotions such as shame and guilt. Participants are asked to rate given behavioral reactions (e.g. "You would think about quitting.") to the presented scenario (e.g. "You break something at work and then hide it.") on a five-point scale, based on how likely they would show the reaction themselves. We used the short version with 11 negative scenarios shown to be equivalent to the long version [43]. For the German version, Cronbach's alpha for the shame-proneness-scale was reported at 0.91 , for the guilt-proneness-scale at 0.57 [44].

The borderline symptom inventory (BSI) is a self-report questionnaire consisting of 52 binary items such as impulsivity, instability of identity and relationships, and depersonalization [45]. Affirmative answers are added up to yield a sum-score between 0 and 52. Internal consistency of the BSI is high (reliability as determined with the Kuder-Richardson-20-Formula is 0.92). The cut-off score of 25 [45] differentiates well between BPD patients and healthy controls (sensitivity $=0.54$, specificity $=0.80$ ) [46]. An un-validated German translation has been published by [47].

\section{MRI data acquisition}

Structural and functional MRI data were acquired using a 3T Philips INGENIA Omega HP scanner (Philips, Best, The Netherlands). Functional images were acquired using a single-shot gradient echo echo-planar imaging (EPI) sequence 
sensitive to blood oxygen level-dependent (BOLD) contrast (repetition time $\mathrm{TR}=2500 \mathrm{~ms}$; echo time $\mathrm{TE}=25 \mathrm{~ms}$; flip angle $=90^{\circ}$; in-plane resolution $=2.5 \times 2.5 \mathrm{~mm}^{2} ; 47$ transversal slices; $2.5 \mathrm{~mm}$ slice thickness; $200 \times 200 \mathrm{~mm}^{2}$ field of view; SENSE factor 2). Additionally, structural images of the whole brain using a T1-weighted 3D TFE sequence $\left(\mathrm{TR}=7.8 \mathrm{~ms}, \mathrm{TE}=3.6 \mathrm{~ms}\right.$, flip angle $8^{\circ}, 1 \times 1 \times 1 \mathrm{~mm}^{3}$ resolution) were acquired.

\section{fMRI preprocessing}

Preprocessing was performed using the SPM12 software package (https://www.fil.ion.ucl.ac.uk/spm/). The preprocessing included the following steps: (i) Correction for differences in the image acquisition time between slices; (ii) a six-parameter rigid body spatial transformation to correct for head motion during data acquisition; (iii) co-registration of the structural image to the mean functional image; (iv) gray and white matter segmentation, bias correction, and spatial normalization of the structural image to a standard template (Montreal Neurological Institute, MNI); (v) spatial normalization of the functional images using the normalization parameters estimated in the previous preprocessing step and resampling to $2.5 \times 2.5 \times 2.5 \mathrm{~mm}^{3}$; (vi) spatial smoothing with a 8-mm full width half maximum Gaussian kernel.

Subjects with strong head motion were excluded from the analysis. The six realignment parameters, i.e., three displacements and three elementary rotations with respect to the first image in the EPI series, were used as an estimator for the head motion. The displacements were required to be smaller than $3.0 \mathrm{~mm}$ (minimum to maximum) and the individual rotations smaller than $3.0^{\circ}$.

\section{Statistical analysis}

Statistical analyses of behavioral data were carried out using IBM $^{\mathrm{TM}}$ Software Package for the Social Sciences (SPSS) ${ }^{\mathrm{TM}}$ for windows, version 23.0.0.1.

Functional images were analyzed using a general linear model. On the single-subject level, a design matrix was defined which included one regressor for each of the four read and four imagine conditions. and the distractor task. Brain activity during each trial was modelled using the canonical hemodynamic response function. The design matrix also included the six motion regressors $(x, y, z$, pitch, roll, yaw) estimated in the head motion correction step during the preprocessing to minimize signal-correlated motion effects. A high-pass filter of $128 \mathrm{~s}$ was applied to the data. Classical parameter estimation was performed with a onelag autoregressive model AR(1) to account for serial correlations in fMRI time series due to aliased biorhythms and un-modelled neuronal activity.
Differences in the processing of shame and guilt scenarios between healthy controls and BPD patients were investigated by a random effects analysis applying voxel-wise two-sample $t$-tests. One-sample $t$-tests were applied to investigate the effect of the experimental manipulation, i.e., brain activation related to the processing of shame and guilt scenarios. To investigate the main effect of shame, guilt, and disgust, we contrasted the imagine phase of those trials with those of the neutral trials. Voxel-wise regression analyses were used to relate changes in regional reactivity with clinical (BSI for BPD patients) and behavioral (TOSCA for patients and controls) data.

Statistical images were assessed for cluster-wise significance using a cluster-defining threshold of $p=0.0001$. A topological family wise error (FWE) procedure was used to correct for multiple comparisons. The $p<0.05$ FWE corrected critical cluster size was $k=45$ voxels. The analysis was performed using SPM12.

\section{Results}

\section{Questionnaire data}

Mean BSI in the patient group was 33.4, which is higher than in other patient groups [45]. Internal consistency of the TOSCA-3 as assessed with Cronbach's $\alpha$ varied between 0.53 (externalization) and 0.92 (shame-proneness). Patients scored higher than controls on the shame-proneness and guilt-proneness subscales, and lower on the subscale detachment. Although groups did not differ in verbal IQ, women with BPD had completed fewer years of school education (see Table 1 for test statistics).

\section{Behavioral results of experimental paradigm}

Mean rates of correct responses in the distraction task were 95.0\% (SD 9.6) in the control group and 95.6\% (SD 9.0) in the BPD group.

\section{Inner tension and dissociation}

Self-reported inner tension during measurements did never exceed $80(M=33.9, \mathrm{SD}=19.9)$, with mean values ranging between 22.5 (before run 1) and 15.7 (after run 3) in the control group (grand mean $=19.0, \mathrm{SD}=10.7$ ) and 49.3 (before run 1) and 54.3 (after run 3) in the BPD group (grand mean $=50.6, \mathrm{SD}=12.7$, see also Fig. $1 \mathrm{~b}$ ). Women with $\mathrm{BPD}$ consistently reported higher inner tension than the control group (two-way mixed ANOVA with repeated measurements: $F(1,38)=68.04, p<0.001)$ There was no main effect for within-subjects factor TIME (Huynh-Feldt-corrected $F(18.4,53.43)=0.54, p=0.569)$, but a GROUP $\times$ TIME 
interaction (Huynh-Feldt-corrected $F(1.84,53.43)=6.61$, $p=0.003)$. Polynomial contrasts revealed a linear decline of inner tension over time in healthy controls $[F(1,16)=4.87$, $p=0.042]$ but a linear rise in BPD patients $[F(1,13)=6.61$, $p=0.023$ ]. In the post measurement questionnaire, all subjects indicated no dissociative symptoms during the measurement.

\section{Vividness of imagination}

Ratings of vividness of imagination were generally high, with mean ratings between $5.5(\mathrm{SD}=2.3)$ and $7.1(\mathrm{SD}=2.4)$ out of 10 for healthy control subjects and BPD patients, respectively. Ratings did not differ between groups but between scenario types, as shown by ANOVA with repeated measurements with between-subjects factor GROUP (control, BPD; $F(1)=0.01, p=0.911$ ) and within-subjects factor TARGET-EMOTION (shame, guilt, disgust, neutral; Greenhouse-Geisser corrected $F(2.42,82.45)=4.50, p=0.009)$. Deviation contrasts revealed below-average vividness ratings in guilt scenarios $[F(1)=9.31, p=0.004]$ and above-average ratings in disgust scenarios $[F(1)=10.38, p=0.003]$.

\section{Intensity of emotions}

Intensity ratings for emotions differed between groups and emotions (Table 2 and Fig. 1c), as shown by three-way mixed ANOVA with within-subjects factors TARGETEMOTION of the scenario (shame, guilt, disgust, neutral; $F(3,90)=11.34, p<0.001)$ and type of EMOTION reported (shame, guilt, disgust, fear, surprise, joy, anger, sadness; Huynh-Feldt corrected $F(4.80,143.96)=22.06$, $p<0.001$ ), and between-subjects factor GROUP (control, BPD; $F(1,30)=7.29, p=0.013)$. Simple contrasts for TARGET-EMOTION showed higher overall emotion intensity in shame and guilt scenarios as compared to neutral scenarios (all $p \leq 0.007$ ). There was a TARGET-EMOTION $\times$ EMOTION interaction (Huynh-Feldt-corrected $F(11.56$,
$347.89)=56.09, p<0.001)$. Pairwise comparisons showed that shame in shame scenarios was rated higher than all other emotions in the same scenario and higher than shame in the other scenarios. The same held true for guilt and disgust (all $p \leq 0.006$ ).

Women with BPD reported higher emotion intensities than healthy women (overall mean difference $=0.96$, $p=0.013)$, particularly for shame and guilt scenarios $[F(3,90)=4.37, p<0.001$ for the GROUP $\times$ TARGET EMOTION interaction; both $p \leq 0.006$ in simple contrasts) and higher reported intensities of shame and guilt in BPD women (Huynh-Feldt-corrected $F(4.80,143.96)=7.78$, $p<0.001$ for the GROUP $\times$ EMOTION interaction; both $p \leq 0.001$ in simple contrasts).

\section{Imaging results}

During the imagine phase in shame trials, subjects showed stronger activations in the right inferior frontal gyrus and the left middle temporal gyrus (Fig. 2a, Table 3a). Imagining guilt-related situations resulted in stronger activations in the superior frontal gyrus and the occipital fusiform gyrus (Fig. 2b, Table 3b). Disgust-related scenarios led to stronger activations in the left inferior frontal gyrus, the left middle temporal gyrus, the right occipital fusiform gyrus, the left anterior insula, the supramarginal gyrus and the superior frontal gyrus (Fig. 2c, Table 3c). When comparing experimental conditions, we found significantly stronger activations in the inferior/middle temporal gyrus contrasting shame versus guilt trials (Table $3 \mathrm{~d}$ ).

In the next step, we examined group differences in the contrasts shame vs. neutral, guilt vs. neutral and disgust vs. neutral. Uncorrected data for between-group effects (guilt vs. neutral and disgust vs. neutral) applying a voxel level threshold of $p<0.001$ and a minimal cluster size of $k=10$ voxels are listed in Supplementary Table 4. At this uncorrected level, guilt scenarios resulted in a higher reactivity in $\mathrm{BPD}$ in the anterior insula, the angular gyrus, the precentral

Table 2 Mean intensity ratings of specific emotions, given separately for groups of participants and types of scenarios

\begin{tabular}{|c|c|c|c|c|c|c|c|c|}
\hline & \multicolumn{2}{|c|}{ Shame scenarios } & \multicolumn{2}{|c|}{ Guilt scenarios } & \multicolumn{2}{|c|}{ Disgust scenarios } & \multicolumn{2}{|c|}{ Neutral scenarios } \\
\hline & Control & BPD & Control & BPD & Control & BPD & Control & BPD \\
\hline Shame & $3.86(0.41)$ & $6.89(0.41)$ & $2.94(0.42)$ & $5.98(0.63)$ & $0.91(0.25)$ & $2.30(0.60)$ & $1.47(0.31)$ & $3.02(0.38)$ \\
\hline Guilt & $0.54(.018)$ & $3.06(0.48)$ & $4.92(0.33)$ & $6.91(0.53)$ & $0.11(0.06)$ & $1.54(0.47)$ & $1.25(0.16)$ & $2.61(0.27)$ \\
\hline Disgust & $0.53(0.21)$ & $2.07(0.47)$ & $0.06(0.06)$ & $0.49(0.25)$ & $4.08(0.50)$ & $5.54(0.49)$ & $0.04(0.04)$ & $0.19(0.12)$ \\
\hline Anger & $2.75(0.38)$ & $3.63(0.60)$ & $2.05(0.48)$ & $3.09(0.69)$ & $0.89(0.24)$ & $1.63(0.50)$ & $0.75(0.31)$ & $1.02(0.27)$ \\
\hline Fear & $0.79(0.28)$ & $2.67(0.58)$ & $1.29(0.33)$ & $2.68(0.67)$ & $0.33(0.19)$ & $1.21(0.46)$ & $0.37(0.13)$ & $1.49(0.37)$ \\
\hline Sadness & $0.92(0.23)$ & $1.96(0.65)$ & $1.80(0.31)$ & $2.79(0.63)$ & $0.14(0.06)$ & $0.86(0.36)$ & $0.37(0.11)$ & $1.47(0.34)$ \\
\hline Joy & $0.41(0.21)$ & $0.09(0.06)$ & $0.32(0.13)$ & $0.23(0.12)$ & $1.27(0.31)$ & $1.46(0.51)$ & $4.26(0.37)$ & $3.21(0.44)$ \\
\hline Surprise & $2.06(0.47)$ & $1.80(0.49)$ & $1.62(0.30)$ & $2.54(0.55)$ & $2.48(0.51)$ & $3.49(0.61)$ & $3.26(0.53)$ & $2.67(0.54)$ \\
\hline
\end{tabular}

Target emotions are printed in bold. Numbers refer to the mean and standard error (in brackets). See text for ANOVA results 
A shame $>$ neutral

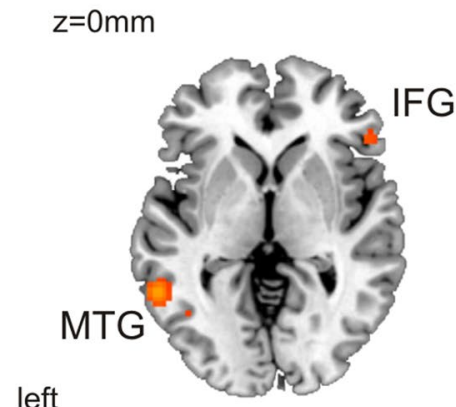

B guilt $>$ neutral

$\mathrm{x}=-5 \mathrm{~mm}$

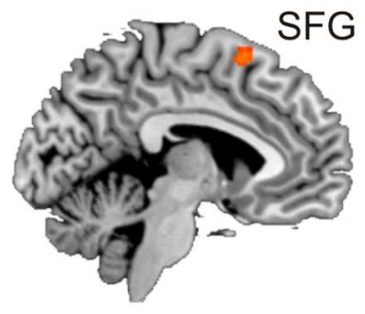

$z=-14 \mathrm{~mm}$

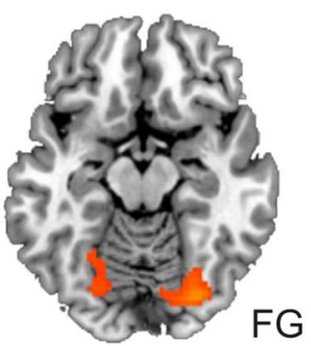

T-value

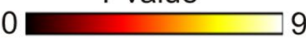

\section{C disgust > neutral}

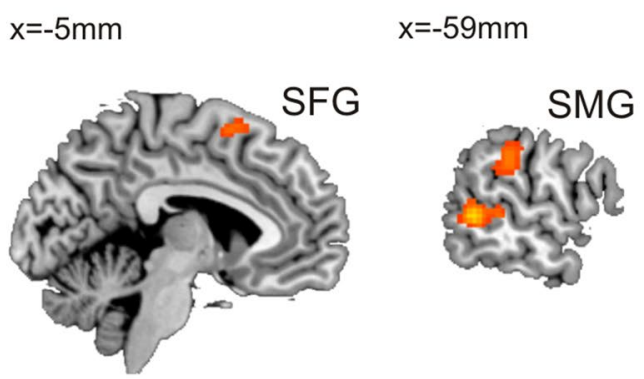

Fig. 2 Neuroimaging results. Brain reactivity to social stimuli during the imagine phase of the task. The results are $p<0.05$ FWE corrected at the cluster level (cluster defining threshold $p=0.0001$ ). a Stronger brain activation during shame compared to neutral scenar-

gyrus and the entorhinal area. No between-group effect at this significance level was found for the contrast shame vs. neutral. Brain activity evoked by emotional stimuli did not correlate with questionnaire data (BSI for BPD patients and TOSCA for patients and controls).

We next tested for group differences specific to the selfconscious emotions shame and guilt. Contrasting shame and guilt scenarios versus disgust scenarios revealed stronger activations in the right amygdala in the BPD group compared to healthy controls $(0.05$ FWE corrected at the cluster level; Fig. 3a, Table 3e). We used cytoarchitectonic probability maps of the amygdala and the hippocampus [48] and the SPM Anatomy toolbox [49] to further specify the anatomical location of our findings. The cluster was mainly located in the superficial and laterobasal nuclear groups of the amygdala. The cluster maximum was assigned to the superficial nuclear group of the amygdala with a probability of $39 \%$. The control group showed no amygdala reactivity for shame ios. b Increased activation for guilt vs. neutral scenarios. c Stronger activation during disgust vs. neutral scenarios. aIns anterior insula, $F G$ fusiform gyrus, $I F G$ inferior frontal gyrus, $M T G$ middle temporal gyrus, $S F G$ superior frontal gyrus

and guilt trials but increased activity for disgust scenarios, whereas the BPD group showed the opposite effect (Fig. 3b).

As the identical scenarios were presented in the three runs, we investigated how the amygdala reactivity evolved over time. Figure $3 \mathrm{c}, \mathrm{d}$ shows the amygdala reactivity contrasting shame and guilt vs. neutral and disgust vs. neutral for each of the three runs separately. We found a significant decline of amygdala reactivity (between first and second run) in healthy controls when imagining shame and guilt scenarios (one-tailed paired $t$-test, $t_{21}=3.133, p=0.003$ ), and disgust scenarios $\left(t_{21}=1.972, p=0.031\right)$. BPD patients, however, showed a significant decline for disgust scenarios (one-tailed paired $t$-test; $t_{18}=1.895, p=0.037$ ), but not for shame and guilt $\left(t_{18}=0.717, p=0.241\right)$. The change in amygdala reactivity between the first and second run did however not reach significance when comparing BPD patients and the HC group (one-tailed two-sample $t$-test; $\left.t_{39}=-1.563, p=0.063\right)$. No further habituation effect for 
Table 3 Imaging results

\begin{tabular}{|c|c|c|c|c|c|c|}
\hline Brain region & Hem & $p$ (FWE) cluster & Cluster size & $p$ (FWE) peak & $T$ peak & MNI coord. (mm) \\
\hline \multicolumn{7}{|l|}{ A) Main effect: shame > neutral } \\
\hline \multirow[t]{2}{*}{ Middle temporal gyrus } & $\mathrm{L}$ & 0.003 & 135 & 0.021 & 5.50 & $-57,-50,0$ \\
\hline & & & & 0.061 & 5.09 & $-47,-64,-5$ \\
\hline \multirow[t]{2}{*}{ Inferior frontal gyrus } & $\mathrm{R}$ & 0.026 & 64 & 0.030 & 5.36 & $48,30,-10$ \\
\hline & & & & 0.240 & 4.51 & $53,28,2$ \\
\hline \multicolumn{7}{|l|}{ B) Main effect: guilt $>$ neutral } \\
\hline Superior frontal gyrus & $\mathrm{L} / \mathrm{R}$ & 0.046 & 45 & 0.039 & 5.31 & $-2,13,60$ \\
\hline \multirow[t]{3}{*}{ Occipital fusiform gyrus } & $\mathrm{R}$ & 0.001 & 148 & 0.039 & 5.31 & $28,-80,-15$ \\
\hline & & & & 0.350 & 4.37 & $10,-74,-12$ \\
\hline & & & & 0.359 & 4.36 & $30,-62,-22$ \\
\hline \multirow[t]{2}{*}{ Occipital fusiform gyrus } & $\mathrm{L}$ & 0.012 & 80 & 0.161 & 4.74 & $-24,-72,-18$ \\
\hline & & & & 0.220 & 4.60 & $-24,-60,-18$ \\
\hline \multicolumn{7}{|l|}{ C) Main effect: disgust $>$ neutral } \\
\hline \multirow[t]{3}{*}{ Inferior frontal gyrus/anterior insula } & $\mathrm{L}$ & $<0.001$ & 217 & $<0.001$ & 8.93 & $-50,28,8$ \\
\hline & & & & 0.010 & 5.82 & $-50,18,8$ \\
\hline & & & & 0.360 & 4.36 & $-44,23,-10$ \\
\hline \multirow[t]{3}{*}{ Middle temporal gyrus } & $\mathrm{L}$ & $<0.001$ & 197 & 0.003 & 6.30 & $-57,-50,2$ \\
\hline & & & & 0.153 & 4.75 & $-60,-37,2$ \\
\hline & & & & 0.221 & 4.59 & $-52,-62,-5$ \\
\hline \multirow[t]{2}{*}{ Occipital fusiform gyrus/cerebellum exterior } & $\mathrm{R}$ & $<0.001$ & 386 & 0.003 & 6.21 & $26,-74,-18$ \\
\hline & & & & 0.120 & 4.86 & $20,-80,-8$ \\
\hline Anterior insula & $\mathrm{L}$ & 0.043 & 47 & 0.019 & 5.58 & $-24,23,-12$ \\
\hline Superior frontal gyrus & $\mathrm{L} / \mathrm{R}$ & 0.018 & 69 & 0.041 & 5.29 & $-2,10,60$ \\
\hline Supramarginal gyrus & $\mathrm{L}$ & 0.013 & 78 & 0.082 & 5.02 & $-62,-30,35$ \\
\hline \multicolumn{7}{|l|}{ D) Main effect: shame > guilt } \\
\hline \multirow[t]{2}{*}{ Middle/inferior temporal gyrus } & $\mathrm{L}$ & 0.014 & 82 & 0.072 & 5.03 & $-54,-52,-10$ \\
\hline & & & & 0.102 & 4.89 & $-52,-60,-8$ \\
\hline \multicolumn{7}{|l|}{ E) BPD $>$ HC: guilt + shame $>$ disgust } \\
\hline Amygdala & $\mathrm{R}$ & 0.047 & 46 & 0.015 & 5.65 & $20,0,-20$ \\
\hline
\end{tabular}

Significant activations for shame $(\mathbf{A})$, guilt $(\mathbf{B})$ and disgust $(\mathbf{C})$ vs. neutral scenarios. D Main effect shame $>$ guilt. E Significant interaction between the group factor and emotional content, i.e., shame and guilt scenarios on the one hand and disgust scenarios on the other hand. The table shows three local maxima more than $8.0 \mathrm{~mm}$ apart

either of the two groups was found comparing the second and third run (all $p>0.05$ ).

\section{Discussion}

The aim of the current study was to investigate the neural basis of altered processing of self-conscious emotions in BPD by instructing participants to imagine fictitious scenarios with shame, guilt, disgust or neutral content. In terms of behavior, the main finding was in agreement with our hypothesis that women with BPD experience more intense feelings when being confronted with affective scenarios, especially higher levels of shame, guilt and fear. With regard to imaging results, we will discuss the main effect of emotional content and then elaborate on differences comparing women with BPD and healthy control subjects. Although we did not observe group differences specific to shame as compared to guilt, we found that women with BPD show generally enhanced amygdala reactivity when experiencing self-conscious emotions. The interaction of group with negative social (shame and guilt) and a negative non-social emotional condition (disgust) is of particular interest here as it allows to partial out effects related to self-relevance and social context rather than emotional valence.

\section{More intense emotional experience in women with BPD}

The participants' ratings demonstrated (i) that the experimental induction of target emotions was successful in both groups, (ii) that women diagnosed with BPD indicated 


\section{A shame+guilt > disgust $x$ GROUP}

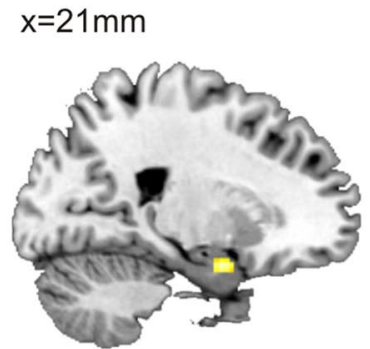

$\mathrm{z}=-21 \mathrm{~mm}$

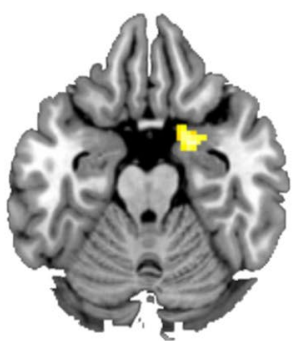

$\mathrm{y}=1 \mathrm{~mm}$

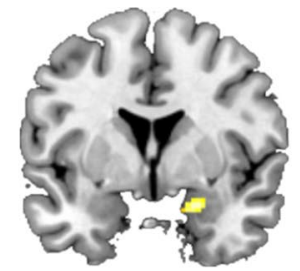

left

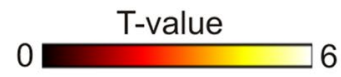

B
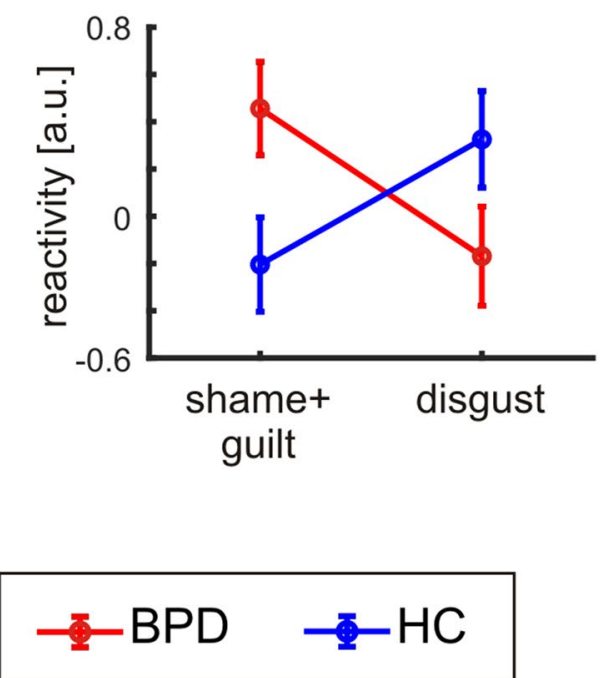

C shame+guilt $>$ neutral

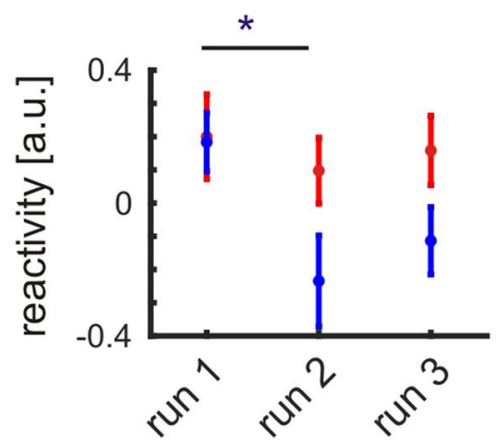

Fig. 3 Group differences in amygdala activation. a Significant interaction between emotional content and group factor in the right amygdala. b The control group shows amygdala activity for disgust scenarios but no reactivity to shame and guilt scenarios, the BPD group shows the opposite effect. c The contrast shame + guilt vs. neutral for

more intense emotional experience, especially of shame and guilt and (iii) that the inner tension increased over time in participants of the BPD group, and decreased in the control group. As a limitation, one might argue that our focus on body shame and avoidance of possible triggers when selecting scenarios might have led participants to experience embarrassment rather than shame. Although rather similar to shame, embarrassment is less associated with morality and has been defined as "an aversive state of mortification, abashment, and chagrin that follows public social predicaments" $[10,50]$. To answer this question, future studies should tap into other aspects of shame and
D disgust $>$ neutral

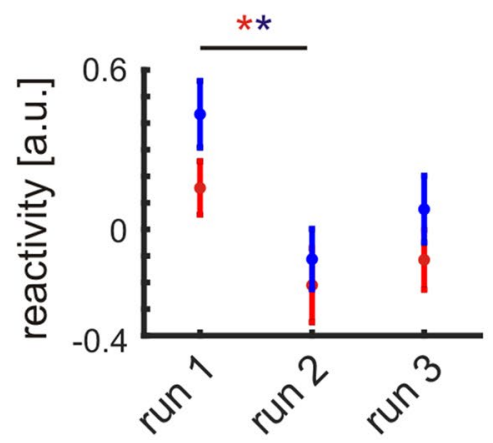

each run separately, showing a habituation effect in $\mathrm{HC}$ but not in the BPD cohort. d Contrasting disgust vs. neutral we find a habituation effect for both HC and the BPD group. The asterisks denote significant differences between the first and the second run

ask for experience of embarrassment in addition to shame and guilt.

\section{Neural correlates of shame and guilt experience}

Contrasting shame, guilt and disgust to neutral scenarios, we identified brain regions previously related to experiencing these emotions $[32,51]$, confirming the validity of our experimental manipulation. These regions are part of the networks implicated more generally in mentalizing and emotion understanding including middle and superior temporal gyrus, anterior insula and adjacent inferior frontal gyrus, 
the precuneus and the temporo-parietal junction [52-56]. Surprisingly, we did not find dorsomedial prefrontal areas and orbitofrontal cortex previously reported in research on self-conscious emotions [32]. Comparing shame and guilt scenarios, we found a significantly higher reactivity for shame scenarios in the posterior part of the medial temporal gyrus. This is in contrast to other studies comparing shame and guilt experience who reported either no shame-specific activation or in other areas [30, 32]. This speaks for the challenge and methodological heterogeneity in studying social emotions which was mentioned already in the introduction, which clearly limits the comparability across studies and thereby the conclusions we can derive about the neural networks specific to shame or guilt experience.

Contrasting emotional to neutral situations, we did not find significant between group effects when correcting for multiple testing. To explore the compatibility of our results with previous and future studies and meta-analyses, we also presented uncorrected results applying a voxel-wise $p$-threshold of $p=0.001$ (Supplementary Table 4). Guilt scenarios resulted in a higher reactivity in BPD in the anterior insula (strongest effect), the angular gyrus, the precentral gyrus and the entorhinal area. In agreement with our finding, an increased activation of the anterior insula for BPD patients contrasting negative emotion and neutral trials was reported in a meta-analysis by Ruocco et al. [35]. The authors argue that an increased activation of the anterior insula might reflect a more intense subjective experience of negative emotion in BPD patients. Thus, whereas the pattern of results is similar to previous findings, we have to assert that based on more rigorous statistical testing, we do not find evidence for general differences in neural activity in women with BPD when experiencing socio-affective situations. The brain activation parameters did not correlate with BPD symptom severity or shame and guilt proneness which might most likely be due to small within-group variance in these measures, probably resulting from our extreme groups design. For future studies it might be interesting to also include a group with subclinical BPD symptoms.

As we will discuss in more detail in the next section, habituation effects play a crucial role in our experiment as the participants were confronted with an identical set of scenarios in each of the three runs. While this allows us to study differences in habituation between BPD patients and control participants, it might lead to decreased sensitivity to overall between-group differences and to the main effect of emotional content due to generally reduced effects in the second and third run.

\section{Reduced habituation to shame and guilt scenarios}

Comparing women with BPD to healthy controls with respect to the self-conscious emotions guilt and shame, we found a significant interaction between emotional content and group in the right amygdala. The control group showed increased amygdala activity in disgust scenarios compared to shame and guilt scenarios. This is in accordance with previous studies in which the amygdala was not involved in processing shame and guilt [32], but in experiencing disgust [51]. The BPD group showed the opposite effect, namely increased amygdala activity for shame and guilt relative to disgust scenarios. In an activation-likelihood-estimation meta-analysis, Ruocco et al. [35] found reduced amygdala activation in BPD patients compared to $\mathrm{HC}$ contrasting negative emotion vs. neutral. In a review article, van Zutphen et al. [33] cited five fMRI studies finding increased amygdala activation in BPD processing emotional stimuli (faces and scenes). As mentioned already in the introduction, numerous explanations have been discussed to account for these inconsistencies including sample characteristics, task methodology, and psychological processes as habituation or dissociation [33, 35]. Addressing these criticisms, we included a non-social emotional condition (disgust), and successfully reduced the risk of dissociation, as neither post measurement questioning nor analysis of the distractor task suggested any dissociation in any of the subjects.

Our results suggest a complex and time-dependent involvement of the amygdala in the processing of emotional stimuli. We interpret the continuously increased amygdala activity in shame and guilt trials as a sign of decreased habituation in BPD, mirrored by a lack of decline in inner tension over the course of the BOLD measurement in this group. This effect was specific when experiencing self-conscious emotions. BPD patients showed the same effect of reduced amygdala activity over time (i.e., experimental runs) as HC when being confronted with disgust scenarios. This supports the notion of habituation differences in the work of van Zutphen et al. [33]. In support of Ruocco et al. [35], amygdala activity in BPD was not increased in the first run of shame and guilt vs. neutral trials. Our results are in line with fMRI results from Hazlett et al. [36]. They reported a potentiated amygdala response to repeatedly shown affective pictures in BPD. Patients exhibited normal amygdala activation during novel pictures but relatively increased amygdala activation during repeated pictures compared with HC. However, the authors used pictures from the IAPS [57], which only consider valence and arousal of the affective stimuli. Moreover, many of the IAPS pictures of negative valence show interpersonal threat or violence, which might trigger traumatic memories in persons with BPD more than in controls. With our study, we extend this work by showing reduced amygdala habituation to specifically self-conscious emotions and using more subtle and less salient stimuli.

From a psychotherapeutic point of view, the lack of amygdala habituation to shame and guilt in BPD patients might relate to the relatively slow improvement in 
emotional reactivity typically seen in therapy [58]. Clinical improvements following psychotherapy have been associated with a normalization of the amygdala response to visual stimuli with aversive affective content [59]. Whether this effect is specific to shame and guilt or extends to other aversive emotions (such as fear) remains to be studied. To date, established psychotherapies for BPD do not focus on the self-conscious emotions [24], but a small pilot study of dialectic behavioral therapy focusing on shame yielded promising results [60]. Our findings attest to the centrality of self-conscious emotions for BPD with more intensive subjective emotional experience and heightened neural response. However, we did not find any evidence for a specificity for shame as compared to guilt and the fMRI findings were overall rather small and clearly need replications with larger samples and other experimental approaches. Moreover, the number of trials for each condition and in each run was rather low as we aimed to use only well-defined scenarios meeting the above-mentioned criteria (see methods section) for our group comparison. While this reduced the heterogeneity across situations, the statistical power to examine condition differences and especially changes across experimental runs was reduced. This again calls for replication with other samples and other experimental approaches to substantiate our findings.

\section{Conclusions}

The central finding in our study was the elevated amygdala reactivity in women with BPD when imagining shame- and guilt-related social scenarios. This was not due to increased activity per se in these scenarios. Rather, a diminished habituation to the presented stimuli across runs reflected an - on average - increased amygdala activity. This effect was specific to guilt and shame as BPD patients showed amygdala habituation comparable to healthy controls to a negative, but not self-conscious emotion (disgust). This finding helps to explain the inconsistencies between previous studies on the involvement of the amygdala in BPD as well as the typically slow progress in the psychotherapy of dysfunctional self-conscious emotions in this patient group.

Acknowledgements Open Access funding provided by Projekt DEAL. We thank all study participants for their valuable time and patience. Also, we thank Christian Erdmann and Dr. Uwe Melchert for conducting the MR measurements and Dr. Valerija Sipos for helpful discussion of the implications of the findings presented here for the psychotherapy of patients with BPD.

Author contributions AW, FB, US and UK designed study, AW and MB collected data, MG and AW analyzed data and prepared first manuscript draft, all authors read and agreed with final draft.

\section{Compliance with ethical standards}

Conflict of interest The authors declare no conflict of interest.

Open Access This article is licensed under a Creative Commons Attribution 4.0 International License, which permits use, sharing, adaptation, distribution and reproduction in any medium or format, as long as you give appropriate credit to the original author(s) and the source, provide a link to the Creative Commons licence, and indicate if changes were made. The images or other third party material in this article are included in the article's Creative Commons licence, unless indicated otherwise in a credit line to the material. If material is not included in the article's Creative Commons licence and your intended use is not permitted by statutory regulation or exceeds the permitted use, you will need to obtain permission directly from the copyright holder. To view a copy of this licence, visit http://creativecommons.org/licenses/by/4.0/.

\section{References}

1. American Psychiatric Association (2013) Diagnostic and statistical manual of mental disorders (DSM-5®). American Psychiatric Pub.

2. Temes CM, Frankenburg FR, Fitzmaurice GM, Zanarini MC (2019) Deaths by suicide and other causes among patients with borderline personality disorder and personality-disordered comparison subjects over 24 years of prospective follow-up. J Clin Psychiatr. https://doi.org/10.4088/jcp.18m12436

\subsection{8/JCP.18m 12436}

3. Leichsenring F, Leibing E, Kruse J, New AS, Leweke F (2011) Borderline personality disorder. Lancet 377(9759):74-84. https:// doi.org/10.1016/S0140-6736(10)61422-5

4. Ellison WD, Rosenstein LK, Morgan TA, Zimmerman M (2018) Community and clinical epidemiology of borderline personality disorder. Psychiatr Clin North Am 41(4):561-573. https://doi. org/10.1016/j.psc.2018.07.008

5. D’Agostino A, Rossi Monti M, Starcevic V (2018) Models of borderline personality disorder: recent advances and new perspectives. Curr Opin Psychiatr 31(1):57-62. https://doi.org/10.1097/ YCO.0000000000000374

6. Sadikaj G, Russell JJ, Moskowitz DS, Paris J (2010) Affect dysregulation in individuals with borderline personality disorder: persistence and interpersonal triggers. J Pers Assess 92(6):490-500. https://doi.org/10.1080/00223891.2010.513287

7. Sauer C, Arens EA, Stopsack M, Spitzer C, Barnow S (2014) Emotional hyper-reactivity in borderline personality disorder is related to trauma and interpersonal themes. Psychiatr Res 220(12):468-476. https://doi.org/10.1016/j.psychres.2014.06.041

8. Tignor SM, Colvin CR (2017) The interpersonal adaptiveness of dispositional guilt and shame: a meta-analytic investigation. J Pers 85(3):341-363. https://doi.org/10.1111/jopy.12244

9. Lewis H (1971) Shame and guilt in neurosis. International Universities Press, New York

10. Tangney JP, Stuewig J, Mashek DJ (2007) Moral emotions and moral behavior. Annu Rev Psychol 58:345-372. https://doi. org/10.1146/annurev.psych.56.091103.070145

11. Joireman J (2004) Empathy and the self-absorption paradox II: self-rumination and self-reflection as mediators between shame, guilt, and empathy. Self Identity 3(3):225-238. https://doi. org/10.1080/13576500444000038 
12. Elison J, Garofalo C, Velotti P (2014) Shame and aggression: theoretical considerations. Aggress Violent Behav 19(4):447-453. https://doi.org/10.1016/j.avb.2014.05.002

13. Stuewig J, Tangney JP, Heigel C, Harty L, McCloskey L (2010) Shaming, blaming, and maiming: functional links among the moral emotions, externalization of blame, and aggression. J Res Pers 44(1):91-102. https://doi.org/10.1016/j.jrp.2009.12.005

14. Niedenthal PM, Tangney JP, Gavanski I (1994) "If only I weren't" versus "if only I hadn't": distinguishing shame and guilt in counterfactual thinking. J Pers Soc Psychol 67(4):585-595. https://doi. org/10.1037//0022-3514.67.4.585

15. Tangney JP, Miller RS, Flicker L, Barlow DH (1996) Are shame, guilt, and embarrassment distinct emotions? J Pers Soc Psychol 70(6):1256-1269. https://doi.org/10.1037//0022-3514.70.6.1256

16. Yang ML, Yang CC, Chiou WB (2010) When guilt leads to other orientation and shame leads to egocentric self-focus: effects of differential priming of negative affects on perspective taking. Soc Behav Pers 38(5):605-614. https://doi.org/10.2224/ sbp.2010.38.5.605

17. Leith KP, Baumeister RF (1998) Empathy, shame, guilt, and narratives of interpersonal conflicts: guilt-prone people are better at perspective taking. J Pers 66(1):1-37

18. Cohen TR, Panter AT, Turan N (2012) Guilt proneness and moral character. Curr Dir Psychol Sci 21(5):355-359. https:// doi.org/10.1177/0963721412454874

19. Brown MZ, Linehan MM, Comtois KA, Murray A, Chapman AL (2009) Shame as a prospective predictor of self-inflicted injury in borderline personality disorder: a multi-modal analysis. Behav Res Ther 47(10):815-822. https://doi.org/10.1016/j. brat.2009.06.008

20. Kim S, Thibodeau R, Jorgensen RS (2011) Shame, guilt, and depressive symptoms: a meta-analytic review. Psychol Bull 137(1):68-96. https://doi.org/10.1037/a0021466

21. Rusch N, Lieb K, Gottler I, Hermann C, Schramm E, Richter H, Jacob GA, Corrigan PW, Bohus M (2007) Shame and implicit self-concept in women with borderline personality disorder. Am J Psychiatr 164(3):500-508. https://doi.org/10.1176/ ajp.2007.164.3.500

22. Gratz KL, Rosenthal MZ, Tull MT, Lejuez CW, Gunderson JG (2010) An experimental investigation of emotional reactivity and delayed emotional recovery in borderline personality disorder: the role of shame. Compr Psychiatr 51(3):275-285. https ://doi.org/10.1016/j.comppsych.2009.08.005

23. Scheel CN, Bender C, Tuschen-Caffier B, Brodführer A, Matthies S, Hermann C, Geisse EK, Svaldi J, Brakemeier E-L, Philipsen A (2014) Do patients with different mental disorders show specific aspects of shame? Psychiatr Res 220(1-2):490-495

24. Karan E, Niesten IJ, Frankenburg FR, Fitzmaurice GM, Zanarini MC (2014) The 16-year course of shame and its risk factors in patients with borderline personality disorder. Personal Ment Health 8(3):169-177. https://doi.org/10.1002/pmh.1258

25. Schoenleber M, Berenbaum H (2012) Shame regulation in personality pathology. J Abnorm Psychol 121(2):433-446. https:// doi.org/10.1037/a0025281

26. Müller-Pinzler L, Krach S, Krämer UM, Paulus FM (2016) The social neuroscience of interpersonal emotions. Social behavior from rodents to humans. Springer, Cham, pp 241-256

27. Muller-Pinzler L, Gazzola V, Keysers C, Sommer J, Jansen A, Frassle S, Einhauser W, Paulus FM, Krach S (2015) Neural pathways of embarrassment and their modulation by social anxiety. NeuroImage 119:252-261. https://doi.org/10.1016/j.neuroimage 2015.06.036

28. Wagner U, N'Diaye K, Ethofer T, Vuilleumier P (2011) Guiltspecific processing in the prefrontal cortex. Cereb Cortex 21(11):2461-2470. https://doi.org/10.1093/cercor/bhr016
29. Takahashi H, Yahata N, Koeda M, Matsuda T, Asai K, Okubo Y (2004) Brain activation associated with evaluative processes of guilt and embarrassment: an fMRI study. NeuroImage 23(3):967974. https://doi.org/10.1016/j.neuroimage.2004.07.054

30. Michl P, Meindl T, Meister F, Born C, Engel RR, Reiser M, Hennig-Fast K (2014) Neurobiological underpinnings of shame and guilt: a pilot fMRI study. Soc Cognit Affect Neurosci 9(2):150157. https://doi.org/10.1093/scan/nss114

31. Pulcu E, Lythe K, Elliott R, Green S, Moll J, Deakin JF, Zahn $R$ (2014) Increased amygdala response to shame in remitted major depressive disorder. PLoS ONE 9(1):e86900. https://doi. org/10.1371/journal.pone.0086900

32. Bastin C, Harrison BJ, Davey CG, Moll J, Whittle S (2016) Feelings of shame, embarrassment and guilt and their neural correlates: a systematic review. Neurosci Biobehav Rev 71:455471. https://doi.org/10.1016/j.neubiorev.2016.09.019

33. van Zutphen L, Siep N, Jacob GA, Goebel R, Arntz A (2015) Emotional sensitivity, emotion regulation and impulsivity in borderline personality disorder: a critical review of fMRI studies. Neurosci Biobehav Rev 51:64-76. https://doi.org/10.1016/j. neubiorev.2015.01.001

34. Linehan MM (1993) Cognitive behavioural therapy of borderline personality disorder. Guilford, New York

35. Ruocco AC, Amirthavasagam S, Choi-Kain LW, McMain SF (2013) Neural correlates of negative emotionality in borderline personality disorder: an activation-likelihood-estimation meta-analysis. Biol Psychiatr 73(2):153-160. https://doi. org/10.1016/j.biopsych.2012.07.014

36. Hazlett EA, Zhang J, New AS, Zelmanova Y, Goldstein KE, Haznedar MM, Meyerson D, Goodman M, Siever LJ, Chu KW (2012) Potentiated amygdala response to repeated emotional pictures in borderline personality disorder. Biol Psychiatr 72(6):448-456. https://doi.org/10.1016/j.biopsych.2012.03.027

37. Wittchen H-U, Zaudig M, Fydrich T (1997) Skid. Strukturiertes klinisches Interview für DSM-IV. Achse I und II. Handanweisung.

38. Philipsen A, Limberger MF, Lieb K, Feige B, Kleindienst N, Ebner-Priemer U, Barth J, Schmahl C, Bohus M (2008) Attention-deficit hyperactivity disorder as a potentially aggravating factor in borderline personality disorder. Br J Psychiatr 192(2):118-123. https://doi.org/10.1192/bjp.bp.107.035782

39. Lehrl S (2005) Mehrfachwahl-Wortschatz-Intelligenztest: MWT-B [Multiple Choice Vocabulary Test, version B]. apitta, Balingen

40. Satzger W, Fessmann H, Engel RR (2002) Liefern HAWIE-R, WST und MWT-B vergleichbare IQ-Werte? (The equivalence of three German vocabulary tests and the German version of the Wechsler Adult Intelligence Scale-Revised (HAWIE-R)). Zeitschrift Differ Diagnos Psychol 23(2):159-170. https://doi. org/10.1024//0170-1789.23.2.159

41. Sipos V, Schweiger U (2017) Treatment of eating disorders by emotion regulation. Kohlhammer Verlag

42. Tangney JP, Dearing R, Wagner PE, Gramzow R (2000) The test of self-conscious affect-3 (TOSCA-3). George Mason University, Fairfax

43. Tangney JP, Dearing R (2002) Shame and guilt. Emotions and social behavior. The Guilford Press, New York

44. Rüsch N, Corrigan PW, Bohus M, Jacob GA, Brueck R, Lieb K (2007) Measuring shame and guilt by self-report questionnaires: a validation study. Psychiatr Res 150(3):313-325

45. Conte HR, Plutchik R, Karasu TB, Jerrett I (1980) A self-report Borderline Scale Discriminative validity and preliminary norms. J Nerv Mental Dis 168(7):428-435

46. Mann LS, Wise TN, Segall EA, Goldberg RL, Goldstein DM (1988) Borderline symptom inventory: assessing inpatient and 
outpatient borderline personality disorders. Psychopathology 21(1):44-50

47. Rohde-Dachser C (2004) Das borderline-syndrom, 7th edn. Huber, Bern Göttingen Toronto Seattle

48. Amunts K, Kedo O, Kindler M, Pieperhoff P, Mohlberg H, Shah NJ, Habel U, Schneider F, Zilles K (2005) Cytoarchitectonic mapping of the human amygdala, hippocampal region and entorhinal cortex: intersubject variability and probability maps. Anat Embryol (Berl) 210(5-6):343-352. https://doi.org/10.1007/s0042 9-005-0025-5

49. Eickhoff SB, Stephan KE, Mohlberg H, Grefkes C, Fink GR, Amunts K, Zilles K (2005) A new SPM toolbox for combining probabilistic cytoarchitectonic maps and functional imaging data. NeuroImage 25(4):1325-1335. https://doi.org/10.1016/j.neuro image.2004.12.034

50. Miller RS (1995) Embarrassment and social behavior. Self-conscious emotions: the psychology of shame, guilt, embarrassment, and pride. Guilford, New York, pp 322-339

51. Schienle A, Stark R, Walter B, Blecker C, Ott U, Kirsch P, Sammer G, Vaitl D (2002) The insula is not specifically involved in disgust processing: an fMRI study. NeuroReport 13(16):2023-2026

52. Van Overwalle F, Baetens K (2009) Understanding others' actions and goals by mirror and mentalizing systems: a meta-analysis. NeuroImage 48(3):564-584. https://doi.org/10.1016/j.neuroimage .2009 .06 .009

53. Van Overwalle F (2009) Social cognition and the brain: a meta-analysis. Hum Brain Mapp 30(3):829-858. https://doi. org/10.1002/hbm.20547
54. Schurz M, Radua J, Aichhorn M, Richlan F, Perner J (2014) Fractionating theory of mind: a meta-analysis of functional brain imaging studies. Neurosci Biobehav Rev 42:9-34. https://doi. org/10.1016/j.neubiorev.2014.01.009

55. Li W, Mai X, Liu C (2014) The default mode network and social understanding of others: what do brain connectivity studies tell us. Front Hum Neurosci 8:74. https://doi.org/10.3389/fnhum .2014 .00074

56. Lieberman MD (2007) Social cognitive neuroscience: a review of core processes. Annu Rev Psychol 58:259-289. https://doi. org/10.1146/annurev.psych.58.110405.085654

57. Lang PJ, Bradley MM, Cuthbert BN (2005) International affective picture system (IAPS): affective ratings of pictures and instruction manual. NIMH, Center for the Study of Emotion \& Attention, Gainesville, Fla.

58. Zanarini MC, Frankenburg FR, Reich DB, Silk KR, Hudson JI, McSweeney LB (2007) The subsyndromal phenomenology of borderline personality disorder: a 10-year follow-up study. A J Psychiatr 164(6):929-935. https://doi.org/10.1176/ajp.2007.164.6.929

59. Links PS, Shah R, Eynan R (2017) Psychotherapy for borderline personality disorder: progress and remaining challenges. Curr Psychiatr Rep 19(3):16. https://doi.org/10.1007/s1192 0-017-0766-x

60. Rizvi SL, Linehan MM (2005) The treatment of maladaptive shame in borderline personality disorder: a pilot study of "opposite action". Cognit Behav Pract 12(4):437-447

61. Oldfield RC (1971) The assessment and analysis of handedness: the Edinburgh inventory. Neuropsychologia 9(1):97-113 